\title{
Multiple benefits from dual release hydrocortisone: a "hard" view from bones
}

\author{
D. A. Vassiliadi ${ }^{1} \cdot$ S. Tsagarakis ${ }^{1}$
}

Received: 9 March 2018 / Accepted: 5 May 2018 / Published online: 17 May 2018

(c) Springer Science+Business Media, LLC, part of Springer Nature 2018

More than 160 years have passed since the description of primary adrenal insufficiency (PAI) by Thomas Addison in 1849 [1] and the only milestone in its management has been the discovery of hydrocortisone. For nearly a century, adrenal insufficiency (AI) was a lethal disease [2]. Hydrocortisone changed the landscape and transformed both PAI and secondary AI (SAI) to a chronic condition $[2,3]$. The fortunate prolongation of survival soon led to the recognition of shortcomings related to hydrocortisone therapy; on the one hand, inadequate replacement, especially during periods of stress, results in life-threatening adrenal crises and, on the other, over-replacement is associated with the adverse effects of hypercortisolism $[4,5]$. As a consequence patients with AI experience poor quality of life, as well as morbidity and mortality rates exceeding those of the general population [4].

As a matter of fact, glucocorticoid replacement remains a suboptimal therapy. Ideally, glucocorticoid administration would mimic diurnal cortisol profiles of healthy people, with higher levels in the morning that decrease toward late evening and night, along with appropriate adjustments during periods of stress. At the same time the total daily dose should be kept to levels corresponding to normal daily cortisol production. Simulating, however, the normal circadian cortisol rhythm with the conventional hydrocortisone preparations is unattainable and even regimens with multiple daily doses are associated with supra-physiological daily peaks [6]. Moreover, data obtained from recent studies showed that the traditional daily dose of $30 \mathrm{mg}$ exceeds considerably the normal rate of cortisol production (approximately $10-20 \mathrm{mg} / \mathrm{day}$ ) [4].

S. Tsagarakis

stsagara@otenet.gr

1 Department of Endocrinology, Diabetes and Metabolism, Evangelismos Hospital, Athens, Greece
The deleterious effects of pharmacological doses of glucocorticoids on bones are well established. The skeletal impact, however, of doses given for glucocorticoid replacement remains somehow controversial, although the bulk of evidence supports an adverse effect $[4,5]$. There is an impressive lack of studies addressing fracture risk in $\mathrm{AI}$ and most data comes from small-scale studies including heterogeneous populations with respect to diagnosis, age, and glucocorticoid regimens [4]. Fracture risk has been reported to be increased, although this association is questioned due to the fact that it increases early after diagnosis and initiation of treatment in PAI, and improves with GH substitution in SAI [4]. Several subpopulations are preferentially affected, such as patients receiving higher doses of glucocorticoids for longer periods of time and those with SAI as compared to PAI [4]. In SAI other hormone deficits, especially GH deficiency and hypogonadism, could also have an adverse effect on bone density and fracture risk, while hydrocortisone over-replacement improves with GH substitution $[4,5]$. Fracture risk could be more important than described to date, particularly regarding vertebral fractures, which are known to be most frequent in glucocorticoidinduced osteoporosis. Morphometric analysis with DEXA demonstrated an increased prevalence of vertebral fractures, three times more than in healthy matched controls, in Addison's patients under a classical of about $30 \mathrm{mg}$ per day dosage of hydrocortisone [7]. This was also the case, in a healthy cohort of post-menopausal women with highest levels of evening endogenous cortisol [8], suggesting that even slight increases in glucocorticoid levels may increase vertebral fracture risk. Thus, fracture risk is probably underestimated as it is not systematically assessed. Loss of bone mass not only is associated with significant morbidity, but also its treatment is problematic; adherence is moderate and there are concerns about side effects. Thus, prevention is preferable and bone health should be considered among the hard end-points when evaluating drawbacks of glucocorticoid replacement. 
Recently, a new dual-release hydrocortisone tablet was developed aiming to achieve a more physiological cortisol profile with an easy, once-daily dosing (Plenadren ${ }^{\circledR}$, Viropharma Inc., Exton, PA, USA). Its tolerance and safety have already been tested. The overall daily cortisol levels are about $20 \%$ lower with this formulation compared to equivalent doses of conventional hydrocortisone and this, in conjunction with its more physiological circadian pattern, might explain its less adverse profile with regard to cardiovascular risk factors, glucose metabolism, and HRQoL compared to treatment with multiple daily hydrocortisone $[9,10]$. In this line, studies focusing on bone effects are of primary importance; so far, data are scanty and in particular patients with SAI were not thoroughly studied.

In this issue of the Journal, Frara et al. [11] report a retrospective observational 24-month study evaluating the dual-release hydrocortisone effects on BMD at lumbar spine, femoral neck, and total hip in 14 patients with SAI who were previously on conventional glucocorticoid replacement regimens. In fact, this is the first study that addresses this issue and only the second longitudinal one on the effects of glucocorticoid substitution on bone health. A characteristic of study population is that the majority had $\mathrm{BMD}$ in the osteoporotic or osteopenic range, despite being on the contemporary lower hydrocortisone dosing. Since this is not a randomized trial, a bias toward selecting patients with worse metabolic or bone profile to switch to Plenadren $^{\circledR}$ cannot be excluded. Over a period of 24 months, BMD increased significantly at lumbar spine and femoral neck but total hip BMD did not change. The median changes in BMD were $+10.0 \%$ at lumbar spine and $+11.5 \%$ at femoral neck, percentages that are comparable to those seen with anti-osteoporotic drugs or in patients withdrawing glucocorticoid therapy. Of note, the other longitudinal study on bone health under glucocorticoid substitution, a prospective one, also showed significant increases in BMD in 27 subjects with PAI, achieved by relatively small reductions in the daily hydrocortisone equivalent dose [12]. Plenadren ${ }^{\circledR}$ probably acts the same way, by reducing the cortisol peaks and the overall daily exposure to hydrocortisone, particularly at late evening and night. The significant increase in bone mineral density was attributed to reversal of the decreased bone resorption followed by increased bone formation, but this remains a hypothesis since bone markers were not studied.

The results of this study are promising, keeping in mind that this is a retrospective cohort study in a relatively small number of patients with SAI. Patients with SAI differ from patients with PAI in that other pituitary hormone deficiencies may also adversely affect skeletal health. The small number of patients, however, did not permit subgroup analysis on concomitant pituitary hormone deficiencies and their management. The present study evaluated BMD as a surrogate marker of fracture risk. However, the relationship between BMD increase and fracture risk reduction is not proportional. Therefore, the magnitude of Plenadren ${ }^{\circledR}$ 's benefit on fracture risk remains to be ascertained.

To summarize, this study represents a step forward for improved formulations to be tested, and hopefully implemented in clinical practice. Plenadren ${ }^{\circledR}$ has a good potential with an easy dosage regimen. So far, this formulation demonstrated a promising metabolic, cardiovascular, healthquality, and immunity-friendly profile compared to the much cheaper traditional hydrocortisone. The present study, showing a clear benefit on bones of patients with SAI, adds further value to its advantageous profile. Until large-scale prospective studies are available, Plenadren ${ }^{\circledR}$ will remain a promising option awaiting more robust evidence for a costeffective role as part of our daily practice. In the meantime, in the absence of biological markers to assess glucocorticoid substitution, it is probably reasonable to propose to patients the lowest glucocorticoid doses allowing good clinical tolerance without increasing the risk for adrenal crisis. The question, however, still remains on the routine need for systematic assessment of BMD as well of radiologic vertebral fractures in this population.

\section{Compliance with ethical standards}

Conflict of interest The authors declare that they have no conflict of interest.

\section{References}

1. P.M. Bishop, The history of the discovery of Addison's disease. Proc. R. Soc. Med. 43(1), 35-42 (1950)

2. D. Dunlop, Eighty-six cases of Addison's disease. Br. Med. J. 2 (5362), 887-891 (1963)

3. S.G. Hillier, Diamonds are forever: the cortisone legacy. J. Endocrinol. 195(1), 1-6 (2007). https://doi.org/10.1677/JOE-070309

4. G. Johannsson, A. Falorni, S. Skrtic, H. Lennernas, M. Quinkler, J.P. Monson, P.M. Stewart, Adrenal insufficiency: review of clinical outcomes with current glucocorticoid replacement therapy. Clin. Endocrinol. 82(1), 2-11 (2015). https://doi.org/10. 1111/cen.12603

5. G. Mazziotti, A.M. Formenti, S. Frara, E. Roca, P. Mortini, A. Berruti, A. Giustina, Management of endocrine disease: risk of overtreatment in patients with adrenal insufficiency: current and emerging aspects. Eur. J. Endocrinol. 177(5), R231-R248 (2017). https://doi.org/10.1530/EJE-17-0154

6. N. Simon, F. Castinetti, F. Ouliac, N. Lesavre, T. Brue, C. Oliver, Pharmacokinetic evidence for suboptimal treatment of adrenal insufficiency with currently available hydrocortisone tablets. Clin. Pharmacokinet. 49(7), 455-463 (2010). https://doi.org/10.2165/ 11531290-000000000-00000

7. V. Camozzi, C. Betterle, A.C. Frigo, V. Zaccariotto, M. Zaninotto, E. De Caneva, P. Lucato, W. Gomiero, S. Garelli, C. Sabbadin, M. Salva, M.D. Costa, M. Boscaro, G. Luisetto, Vertebral fractures assessed with dual-energy X-ray absorptiometry in patients with Addison's disease on glucocorticoid and mineralocorticoid 
replacement therapy. Endocrine 59(2), 319-329 (2018). https:// doi.org/10.1007/s12020-017-1380-8

8. E. Gonzalez Rodriguez, O. Lamy, D. Stoll, M. Metzger, M. Preisig, C. Kuehner, P. Vollenweider, P. Marques-Vidal, G. Waeber, B. Aubry-Rozier, D. Hans, High evening cortisol level is associated with low TBS and increased prevalent vertebral fractures: OsteoLaus study. J. Clin. Endocrinol. Metab. 102(7), 2628-2636 (2017). https://doi.org/10.1210/jc.2016-3804

9. G. Johannsson, A.G. Nilsson, R. Bergthorsdottir, P. Burman, P. Dahlqvist, B. Ekman, B.E. Engstrom, T. Olsson, O. Ragnarsson, M. Ryberg, J. Wahlberg, B.M. Biller, J.P. Monson, P.M. Stewart, H. Lennernas, S. Skrtic, Improved cortisol exposure-time profile and outcome in patients with adrenal insufficiency: a prospective randomized trial of a novel hydrocortisone dual-release formulation. J. Clin. Endocrinol. Metab. 97(2), 473-481 (2012). https:// doi.org/10.1210/jc.2011-1926
10. M. Quinkler, R. Miodini Nilsen, K. Zopf, M. Ventz, M. Oksnes, Modified-release hydrocortisone decreases BMI and HbA1c in patients with primary and secondary adrenal insufficiency. Eur. J. Endocrinol. 172(5), 619-626 (2015). https://doi.org/10.1530/EJE14-1114

11. S. Frara, S. Chiloiro, T. Porcelli, A. Giampietro, G. Mazziotti, L. De Marinis, A. Giustina, Bone safety of dual-release hydrocortisone in patients with hypopituitarism. Endocrine (2018). https://doi.org/10.1007/s12020-017-1512-1

12. J. Schulz, K.R. Frey, M.S. Cooper, K. Zopf, M. Ventz, S. Diederich, M. Quinkler, Reduction in daily hydrocortisone dose improves bone health in primary adrenal insufficiency. Eur. J. Endocrinol. 174(4), 531-538 (2016). https://doi.org/10.1530/EJE15-1096 